\title{
Global Regularity Criterion for the 3D Incompressible Navier-Stokes Equations Involving the Velocity Partial Derivative
}

\author{
TianLi Li $\mathbb{D}^{1}$ and Wen Wang $\mathbb{D}^{2}$ \\ ${ }^{1}$ Department of Basic Education, Anhui Vocational and Technical College, Hefei 230011, China \\ ${ }^{2}$ School of Mathematics and Statistics, Hefei Normal University, Hefei 230601, China \\ Correspondence should be addressed to TianLi Li; litianli87423@163.com and Wen Wang; wwen2014@mail.ustc.edu.cn
}

Received 7 January 2021; Revised 30 April 2021; Accepted 19 May 2021; Published 2 June 2021

Academic Editor: Luigi Rodino

Copyright (c) 2021 TianLi Li and Wen Wang. This is an open access article distributed under the Creative Commons Attribution License, which permits unrestricted use, distribution, and reproduction in any medium, provided the original work is properly cited.

In this paper, we study the regularity of the weak solutions for the incompressible 3D Navier-Stokes equations with the partial derivative of the velocity. By the embedded technology, we prove that the weak solution $u$ is regular on $(0, T]$ if $\partial_{3} u \in L^{p}\left(0, T ; L^{q}\left(R^{3}\right)\right)$ with $(2 / p)+(3 / q)=(70 / 37)+(15 / 37 q),(15 / 4) \leq q \leq \infty$, or $(2 / p)+(3 / q)=(34 / 19)+(9 / 19 q),(9 / 4)$ $\leq q \leq \infty$.

\section{Introduction and the Main Result}

This paper focuses on the following three-dimensional incompressible Navier-Stokes (N-S) equations:

$$
\left\{\begin{array}{l}
u_{t}+u \cdot \nabla u+\nabla p=\Delta u, \\
\nabla \cdot u=0, \\
u(x, 0)=u_{0}(x),
\end{array}\right.
$$

where $u$ and $p$ denote the velocity field and the pressure, respectively, and $u_{0}(x)$ is the initial fluid which satisfied $\nabla \cdot u_{0}=0$.

The existence of weak solutions of N-S equations was proved by Leray [1] and Hopf [2]. However, the existence of 3D global regular solutions is still an open question. Prodi [3] and Serrin [4] first considered the regularity of solutions. They, respectively, proved that the weak solution of $3 \mathrm{D} \mathrm{N}-\mathrm{S}$ equations is regular when the exponents $p$ and $q$ satisfy

$$
u \in L^{p}\left(0, T ; L^{q}\left(R^{3}\right)\right)=L_{t}^{p} L_{x}^{q}, \frac{2}{p}+\frac{3}{q}=1, \quad 3 \leq q \leq \infty .
$$

In 1995, Veiga [5] generalized the result to

$$
\nabla u \in L_{t}^{p} L_{x}^{q}, \frac{2}{p}+\frac{3}{q}=2, \quad \frac{3}{2} \leq q \leq \infty .
$$

When $\nabla u, \nabla u_{3}, u_{3}$, and the like satisfy a certain integrable condition, the weak solution is regular, and a large number of results are obtained (for details, refer to [6-16]). And Penel and Pokorný [13], Kukavica and Ziane [14], Cao [15], and Zhang [16], respectively, proved that the weak solution is regular on $(0, T]$ when the weak solution satisfies the following conditions:

$$
\begin{array}{ll}
\partial_{3} u \in L_{t}^{p} L_{x}^{q}, \frac{2}{p}+\frac{3}{q}=\frac{3}{2}, \quad 2 \leq q \leq \infty, \\
\partial_{3} u \in L_{t}^{p} L_{x}^{q}, \frac{2}{p}+\frac{3}{q}=2, \quad \frac{9}{4} \leq q \leq 3, \\
\partial_{3} u \in L_{t}^{p} L_{x}^{q}, \frac{2}{p}+\frac{3}{q}=2, \quad \frac{27}{16} \leq q \leq \frac{5}{2}, \\
\partial_{3} u \in L_{t}^{p} L_{x}^{q}, \frac{2}{p}+\frac{3}{q}=2, \quad 1.56 \approx \frac{3 \sqrt{37}}{4}-3 \leq q \leq 3 .
\end{array}
$$

Recently, Zhang, Yuan, and Zhou in [17] proved if

$$
\partial_{3} u \in L_{t}^{p} L_{x}^{q}, \frac{2}{p}+\frac{3}{q}=\frac{8}{5}+\frac{3}{5 q} \leq 1.75, \quad 4 \leq q \leq \infty,
$$


$\partial_{3} u \in L_{t}^{p} L_{x}^{q}, \frac{2}{p}+\frac{3}{q}=\frac{14}{11}+\frac{9}{11 q} \leq 1.6, \quad \frac{5}{2} \leq q \leq \infty$,

the weak solution is regular on $(0, T]$.

Very recently, LI and Dong in [18] proved if

$\partial_{3} u \in L_{t}^{p} L_{x}^{q}, \frac{2}{p}+\frac{3}{q}=\frac{46}{25}+\frac{3}{25 q} \leq 1.871, \quad \frac{31}{8} \leq q \leq \infty$,

or

$$
\partial_{3} u \in L_{t}^{p} L_{x}^{q}, \frac{2}{p}+\frac{3}{q}=\frac{22}{13}+\frac{3}{13 q} \leq 1.789, \quad \frac{19}{8} \leq q \leq \infty,
$$

the weak solution is regular on $(0, T]$.

One of our main tasks is that when the value of the equation is as constant as possible, we expand the range of $q$ to make the value of $q$ as small as possible. Second, when the range of $q$ does not shrink, the equation is tried to enlarge. Inspired by the texts $[14,17]$, we get better results than the above [17] as follows.

Theorem 1. Assume $u_{0} \in L^{2}\left(\mathbb{R}^{3}\right)$ and $\nabla \cdot u_{0}=0, T>0$. Let $u$ be a weak solution of $N$-S equations (1) on $(0, T]$ which satisfies the initial value of $u_{0}$. If

$$
\partial_{3} u \in L_{t}^{p} L_{x}^{q}, \frac{2}{p}+\frac{3}{q}=\frac{70}{37}+\frac{15}{37 q} \leq 2, \quad \frac{15}{4} \leq q \leq \infty,
$$

then the weak solution is regular on $(0, T]$.

Theorem 2. Assume $u_{0} \in L^{2}\left(\mathbb{R}^{3}\right)$ and $\nabla \cdot u_{0}=0, T>0$. Let $u$ be a weak solution of $N$-S equations (1) on $(0, T]$ which satisfies the initial value of $u_{0}$. If

$$
\partial_{3} u \in L_{t}^{p} L_{x}^{q}, \frac{2}{p}+\frac{3}{q}=\frac{34}{19}+\frac{9}{19 q} \leq 2, \quad \frac{9}{4} \leq q \leq \infty,
$$

then the weak solution is regular on $(0, T]$.

Remark 1. Comparing (9) and (7) and (10) and (8), respectively, the value of $q$ is reduced, and within the range of $q$, the value of the equation is larger than that, so it is better. And when $q$ obtains the minimum value, respectively, each equation takes the critical value of 2 , so this condition is the optimal critical value.

However, we find that, in the existing results, the maximum value of $q$ is bounded when the equality value can reach 2 . The highlight of this article is that the equation value goes to 2 , and the $q$ value reaches infinity. When the value of the equation reached 2 , it is a difficult problem to prove the regularity of the weak solutions to the $3 \mathrm{D}$ incompressible $\mathrm{N}-\mathrm{S}$ equations by adding the value of $q$ getting to infinity and getting to the current minimum of $(3 \sqrt{37} / 4)-3$. We hope we can overcome this problem in the near future.
We shall give the proof of our main result in the third part. In order to facilitate reading, we will give the necessary preparatory knowledge in the following section.

\section{Preliminaries}

Throughout this text, $C$ stands for a generic positive constant which may differ in value from one line to another. We use $\|\cdot\|_{L^{p}}$ to denote the norm of the Lebesgue space $L^{p}(1 \leq p \leq \infty)$ as follows:

$$
\|f\|_{L^{P}}= \begin{cases}\left(\int_{R^{3}}|f(x)|^{p} \mathrm{~d} x\right)^{(1 / p)}, & 1 \leq p<\infty \\ \underset{x \in R^{3}}{\operatorname{esssup}}|f(x)|, & p=\infty .\end{cases}
$$

Definition 1 (see [19]). Assume $u_{0} \in L^{2}\left(\mathbb{R}^{3}\right)$ and $\nabla \cdot u_{0}=0, T>0$. The measurable function $u$ defined on $(0, T] \times R^{3}$ is called the weak solution of equation (1) if

(1) $u \in L^{\infty}\left(0, T ; L^{2}\left(R^{3}\right)\right) \cap L^{2}\left(0, T ; H^{1}\left(R^{3}\right)\right)$.

(2) $\nabla \cdot u=0$ and $\forall \varphi \in C_{0}^{\infty}\left((0, T) \times R^{3}\right)$ satisfy

$$
\int_{0}^{T} \int_{R^{3}} u \cdot \nabla \varphi \mathrm{d} x \mathrm{~d} t=0
$$

and equation (1) holds in the sense of distributions. For $\forall \varphi \in C_{0}^{\infty}\left((0, T) \times R^{3}\right)$ and $\nabla \cdot \varphi=0$ satisfy

$$
\begin{aligned}
& \int_{0}^{T} \int_{R^{3}}\left(\partial_{t} \varphi+(u \cdot \nabla) \varphi\right) \cdot u \mathrm{~d} x \mathrm{~d} t+\int_{R^{3}} u_{0} \cdot \varphi(x, 0) \mathrm{d} x \\
& \quad=\int_{0}^{T} \int_{R^{3}} \nabla u: \nabla \varphi \mathrm{d} x \mathrm{~d} t,
\end{aligned}
$$

where $A: B=\sum_{i, j=1}^{3} a_{i j} b_{i j}, \quad A=\left(a_{i j}\right), B=\left(b_{i j}\right)$.

(3) The strong energy inequality, that is,

$$
\|u(t)\|_{L^{2}}^{2}+2 \int_{0}^{t}\|\nabla u(\tau)\|_{L^{2}}^{2} d \tau \leq\|u(0)\|_{L^{2}}^{2}, \quad \forall 0<t<T .
$$

Lemma 1 (see [13]).

$$
\|p\|_{L^{r}} \leq C\|u\|_{L^{2} r}^{2}
$$

$\left\|\partial_{3} p\right\|_{L^{r}} \leq C \sum_{i, j=1}^{3}\left\|\partial_{3}\left(u_{i} u_{j}\right)\right\|_{L^{r}}, \quad 1<r<\infty$.

Lemma 2 (Sobolev embedding inequality).

$$
\|u\|_{L^{3 r}} \leq C\left\|\partial_{1} u\right\|_{L^{2}}^{(1 / 3)}\left\|\partial_{2} u\right\|_{L^{2}}^{(1 / 3)}\left\|\partial_{3} u\right\|_{L^{r}}^{(1 / 3)}, \quad 1<r \leq \infty .
$$




\section{Proof of Main Results}

In this part, we give the proof of main results. In order to prove Theorems 1 and 2, we thank the results in [13]. In [13], Penel and Pokorný showed that if

$$
\begin{aligned}
& u_{3} \in L^{(2 s /(s-3))}\left(0, T ; L^{s}\left(R^{3}\right)\right), \quad 3<s \leq \infty, \\
& \partial_{3} u_{1}, \partial_{3} u_{1} \in L^{(2 q /(2 q-3))}\left(0, T ; L^{s}\left(R^{3}\right)\right), \quad \frac{3}{2}<q \leq \infty,
\end{aligned}
$$

then the weak solution of the N-S equations is regular on $(0, T]$.

For arbitrary $(15 / 4) \leq q \leq \infty$ and $(9 / 4) \leq q \leq \infty$, we have

$$
\begin{aligned}
& L^{(37 q /(35 q-48))}\left(0, T ; L^{q}\left(R^{3}\right)\right) \subset L^{(2 q /(2 q-3))}\left(0, T ; L^{q}\left(R^{3}\right)\right), \\
& L^{(19 q /(17 q-24))}\left(0, T ; L^{q}\left(R^{3}\right)\right) \subset L^{(2 q /(2 q-3))}\left(0, T ; L^{q}\left(R^{3}\right)\right),
\end{aligned}
$$

respectively.
Hence, they just have to prove that

$$
\begin{array}{ll}
\partial_{3} u \in L^{p}\left(0, T ; L^{q}\left(R^{3}\right)\right), \frac{2}{p}+\frac{3}{q}=\frac{70}{37}+\frac{15}{37 q}, & \frac{15}{4} \leq q \leq \infty, \\
\partial_{3} u \in L^{p}\left(0, T ; L^{q}\left(R^{3}\right)\right), \frac{2}{p}+\frac{3}{q}=\frac{34}{19}+\frac{9}{19 q}, \quad \frac{9}{4} \leq q \leq \infty,
\end{array}
$$

and in both cases, $u_{3} \in L^{(2 s /(s-3))}\left(0, T ; L^{s}\left(R^{3}\right)\right), \quad 3<s \leq \infty$, is established.

Taking the inner product for $u_{3}$ by $\left|u_{3}\right| u_{3}$ and integrating over $R^{3}$, we have

$$
\frac{1}{3} \frac{\mathrm{d}}{\mathrm{d} t}\left\|u_{3}\right\|_{L^{3}}^{3}+\frac{4}{9}\left\|\nabla\left|u_{3}\right|^{(3 / 2)}\right\|_{L^{2}}^{2}=-\int_{R^{3}} \partial_{3} p\left|u_{3}\right| u_{3} \mathrm{~d} x=I,
$$

where we use $\nabla \cdot u=0$ and the following identities:

$$
\begin{aligned}
\int_{R^{3}} u \cdot \nabla u_{3}\left|u_{3}\right| u_{3} \mathrm{~d} x & =\frac{1}{3} \int_{R^{3}} u \cdot \nabla\left|u_{3}\right|^{3} \mathrm{~d} x \\
& =\frac{1}{3} \int_{R^{3}} \nabla \cdot u\left|u_{3}\right|^{3} \mathrm{~d} x=0, \\
-\int_{R^{3}} \Delta u_{3}\left|u_{3}\right| u_{3} \mathrm{~d} x & =\int_{R^{3}}\left(\nabla u_{3}\right) \cdot \nabla\left|u_{3}\right| u_{3} \mathrm{~d} x+\int_{R^{3}}\left(\nabla u_{3}\right) \cdot \nabla u_{3}\left|u_{3}\right| \mathrm{d} x \\
& =\frac{1}{2} \int_{R^{3}}\left(\nabla\left|u_{3}\right|^{2}\right) \cdot \nabla\left|u_{3}\right| \mathrm{d} x+\int_{R^{3}}\left|\nabla u_{3}\right|^{2}\left|u_{3}\right| \mathrm{d} x \\
& =\frac{4}{9} \int_{R^{3}}\left(\nabla\left|u_{3}\right|^{(3 / 2)}\right)^{2} \mathrm{~d} x+\int_{R^{3}}\left|\nabla u_{3}\right|^{2}\left|u_{3}\right| \mathrm{d} x \\
& \geq \frac{4}{9} \int_{R^{3}}\left(\nabla\left|u_{3}\right|^{(3 / 2)}\right)^{2} \mathrm{~d} x .
\end{aligned}
$$

Proof of Theorem 1. We estimate the right side of (21). By using the Hölder inequality, the Young inequality, (14), (16), and (17), we get that

$$
\begin{aligned}
|I| \leq C & \left\|\partial_{3} p\right\|_{L^{3}}\left\|u_{3}\right\|_{L^{3}}^{2} \\
\leq & C\left\|u \partial_{3} u\right\|_{L^{3}}\left\|u_{3}\right\|_{L^{3}}^{2} \\
\leq & C\|u\|_{L^{(3 q /(q-3))}}\left\|\partial_{3} u\right\|_{L^{q}}\left\|u_{3}\right\|_{L^{3}}^{2} \\
\leq & C\|u\|_{L^{2}}^{((4 q-15) /(2(3 q-2)))}\|u\|_{L^{3 q}}^{((q+24) /(4(3 q-2)))}\left\|\partial_{3} u\right\|_{L^{2}}\left\|u_{3}\right\|_{L^{3}}^{2} \\
\leq & C\left\|\partial_{1} u\right\|_{L^{2}}^{((q+24) /(12(3 q-2)))}\left\|\partial_{2} u\right\|_{L^{2}}^{((q+24) /(12(3 q-2)))} \\
& \cdot\left\|\partial_{3} u\right\|_{L^{q}}^{((q+24) /(12(3 q-2)))}\left\|\partial_{3} u\right\|_{L^{q}}\left\|u_{3}\right\|_{L^{3}}^{2} \\
\leq & C\|\nabla u\|_{L^{2}}^{(q+24 /(6(3 q-2)))}\left\|\partial_{3} u\right\|_{L^{q}}^{(37 q /(12(3 q-2)))}\left\|u_{3}\right\|_{L^{3}}^{2} \\
\leq & C\left(\|\nabla u\|_{L^{2}}^{2}+\left\|\partial_{3} u\right\|_{L^{q}}^{(37 q /(35 q-48))}\right)\left\|u_{3}\right\|_{L^{3}}^{2} .
\end{aligned}
$$

Substituting (23) into (21), we have

$$
\frac{\mathrm{d}}{\mathrm{d} t}\left\|u_{3}\right\|_{L^{3}}^{3} \leq C\left(\|\nabla u\|_{L^{2}}^{2}+\left\|\partial_{3} u\right\|_{L^{q}}^{(37 q /(35 q-48))}\right)\left\|u_{3}\right\|_{L^{3}}^{2} .
$$

Dividing both sides by $\left\|u_{3}\right\|_{L^{3}}^{2}$ and integrating with respect to $t$ imply that

$$
\int_{0}^{T} d\left\|u_{3}\right\|_{L^{3}} \leq C \int_{0}^{T}\left(\|\nabla u\|_{L^{2}}^{2}+\left\|\partial_{3} u\right\|_{L^{q}}^{(37 q /(35 q-48))}\right) \mathrm{d} t .
$$

We deduce from (9) and (14) that

$$
\left\|u_{3}\right\|_{L^{\infty}\left(0, T ; L^{3}\left(R^{3}\right)\right)} \leq C .
$$

It is available from type (23) that

$$
\frac{4}{9}\left\|\nabla\left|u_{3}\right|^{(3 / 2)}\right\|_{L^{2}}^{2} \leq C\left(\|\nabla u\|_{L^{2}}^{2}+\left\|\partial_{3} u\right\|_{L^{q}}^{(37 q /(35 q-48))}\right)\left\|u_{3}\right\|_{L^{3}}^{2} \text {. }
$$


So, by the embedding inequality, we get

$$
\begin{aligned}
\left\|u_{3}\right\|_{L^{3}\left(0, T ; L^{9}\left(R^{3}\right)\right)} & =\left\|\left|u_{3}\right|^{(3 / 2)}\right\|_{L^{2}\left(0, T ; L^{6}\left(R^{3}\right)\right)}^{(2 / 3)} \\
& \leq C\left\|\nabla\left|u_{3}\right|^{(3 / 2)}\right\|_{L^{2}\left(0, T ; L^{2}\left(R^{3}\right)\right)}^{(2 / 3)} \leq C .
\end{aligned}
$$

So, Theorem 1 is proved.

Proof of Theorem 2. We have another estimation for the right side of (21). By using the Hölder inequality, the Young inequality, (14), (15), and (17), we have

$$
\begin{aligned}
|I| \leq & C \int_{R^{3}}|p|\left|\partial_{3} u_{3} \| u_{3}\right| \mathrm{d} x \\
\leq & C\|p\|_{L^{(3 q /(2 q-3))}}\left\|\partial_{3} u_{3}\right\|_{L^{q}}\left\|u_{3}\right\|_{L^{3}} \\
\leq & C\|u\|_{L^{(6 q /(2 q-3))}}^{2}\left\|\partial_{3} u\right\|_{L^{q}}\left\|u_{3}\right\|_{L^{3}} \\
\leq & C\|u\|_{L^{2}}^{((4 q-9) /(3(3 q-2)))}\|u\|_{L^{3 q}}^{((q+12) /(2(3 q-2)))}\left\|\partial_{3} u\right\|_{L^{q}}\left\|u_{3}\right\|_{L^{3}} \\
\leq & C\left\|\partial_{1} u\right\|_{L^{2}}^{((q+12) /(6(3 q-2)))}\left\|\partial_{2} u\right\|_{L^{2}}^{((q+12) /(6(3 q-2)))} \\
& \cdot\left\|\partial_{3} u\right\|_{L^{q}}^{((q+12) /(6(3 q-2)))}\left\|\partial_{3} u\right\|_{L^{q}}\left\|u_{3}\right\|_{L^{3}} \\
\leq & C\|\nabla u\|_{L^{2}}^{((q+12) /(3(3 q-2)))}\left\|\partial_{3} u\right\|_{L^{q}}^{(19 q /(6(3 q-2)))}\left\|u_{3}\right\|_{L^{3}} \\
\leq & C\left(\|\nabla u\|_{L^{2}}^{2}+\left\|\partial_{3} u\right\|_{L^{q}}^{(19 q /(17 q-24))}\right)\left\|u_{3}\right\|_{L^{3}} .
\end{aligned}
$$

Inserting (29) into (21), one has

$$
\frac{\mathrm{d}}{\mathrm{d} t}\left\|u_{3}\right\|_{L^{3}}^{3} \leq C\left(\|\nabla u\|_{L^{2}}^{2}+\left\|\partial_{3} u\right\|_{L^{q}}^{(19 q /(17 q-24))}\right)\left\|u_{3}\right\|_{L^{3}}
$$

Dividing both sides by $\left\|u_{3}\right\|_{L^{3}}$ and integrating with respect to $t$ imply that

$$
\int_{0}^{T}\left\|u_{3}\right\|_{L^{3}} d\left\|u_{3}\right\|_{L^{3}} \leq C \int_{0}^{T}\left(\|\nabla u\|_{L^{2}}^{2}+\left\|\partial_{3} u\right\|_{L^{q}}^{(19 q /(17 q-24))}\right) \mathrm{d} t
$$

By (10) and (14), we have

$$
\left\|u_{3}\right\|_{L^{\infty}\left(0, T ; L^{3}\left(R^{3}\right)\right)} \leq C .
$$

The same can be proved:

$$
u_{3} \in L^{3}\left(0, T ; L^{9}\left(R^{3}\right)\right)
$$

So, Theorem 2 is proved.

\section{Data Availability}

No data were used to support this study.

\section{Conflicts of Interest}

The authors declare that they have no conflicts of interest.

\section{Acknowledgments}

Li was supported by the Anhui Provincial Natural Science Fund Project (KJ2018B0002). Wang was supported by the NSF of Anhui Province (1908085QA04).

\section{References}

[1] L. Sur, "Le mouvement dun liquide visqueux emplissant lespace," Acta Mathematica, vol. 63, pp. 193-248, 1934, (In French)

[2] E. Hopf, "Uber die Anfangswertaufgabe Fur die hydrodynamischen Grundgleichungen," Mathematische Nachrichten, vol. 4, pp. 213-231, 1951, (In German).

[3] G. Prodi, "Un teorema di unicità per le equazioni di NavierStokes," Annali di Matematica Pura ed Applicata, vol. 48, no. 1, pp. 173-182, 1959.

[4] J. Serrin, "The initial value problems for the Navier-Stokes," in Nonlinear Problems, R. E. Langer, Ed., 1963.

[5] B.-D. Veiga, "A new regularity class for the Navier-Stokes equations," Chinese Annals of Mathematics, Series B, vol. 407, pp. 1-6, 1995.

[6] M. Pokorn, "On the result of He concerning the smoothness of solutions to the Navier-Stokes equations," Electronic Journal of Differential Equations, vol. 11, p. 1, 2003.

[7] Y. Zhou, "A new regularity criterion for the Navier-Stokes equations in terms of the gradient of one velocity component," Methods and Applications of Analysis, vol. 9, no. 4, pp. 563578, 2002.

[8] Y. Zhou, "A new regularity criterion for weak solutions to the Navier-Stokes equations," Journal de Mathématiques Pures et Appliquées, vol. 84, no. 11, pp. 1496-1514.

[9] C. Cao and E. S. Titi, "Regularity criteria for the three-dimensional Navier-Stokes equations," Indiana University Mathematics Journal, vol. 57, no. 6, pp. 2643-2662, 2008.

[10] B.-Q. Dong and Z.-M. Chen, "Regularity criterion of weak solutions to the $3 \mathrm{D}$ Navier-Stokes equations via two velocity components," Journal of Mathematical Analysis and Applications, vol. 338, no. 1, pp. 1-10, 2008.

[11] J. Wolf, "A regularity criterion of Serrin-type for the NavierStokes equations involving the gradient of one velocity component," Analysis, vol. 5, pp. 259-292, 2015.

[12] Z. Guo, M. Caggio, and Z. Skalák, "Regularity criteria for the Navier-Stokes equations based on one component of velocity," Nonlinear Analysis: Real World Applications, vol. 35, pp. 379-396, 2017.

[13] P. Penel and M. Pokorný, "Some new regularity criteria for the Navier-Stokes equations containing gradient of the velocity," Applications of Mathematics, vol. 49, no. 5, pp. 483-493, 2004.

[14] I. Kukavica and M. Ziane, "Navier-Stokes equations with regularity in one direction," Journal of Mathematical Physics, p. $48,2007$.

[15] C. Cao, "Sufficient conditions for the regularity to the $3 \mathrm{D}$ Navier-Stokes equations," Discrete \& Continuous Dynamical Systems - A, vol. 26, no. 4, pp. 1141-1151, 2010.

[16] Z. Zhang, "An improved regularity criterion for the NavierStokes equations in terms of one directional derivative of the velocity field," Bulletin of Mathematical Sciences, vol. 8, no. 1, pp. 33-47, 2018.

[17] Z. Zhang, W. Yuan, and Y. Zhou, "Some remarks on the Navier-Stokes equations with regularity in one direction," Applications of Mathematics, vol. 64, no. 3, pp. 301-308, 2019. 
[18] T.-L. LI and B.-Q. Dong, "Global regularity criterion for the 3D incompressible Navier-Stokes equations," College Mathematics, vol. 36, pp. 1-6, 2020, (in Chinese).

[19] P.-G. Lemarie-Rieussset, Recent Developments in the NavierStokes Problem, Chapman \& Hall, London, 2002. 